\title{
A prototype vector magnetic field monitoring system for a neutron electric dipole moment experiment
}

\section{N. Nouri ${ }^{a *}$, A. Biswas ${ }^{b}$, M. A. Brown ${ }^{a}$, R. Carr $^{b}$, B. Filippone $^{b}$, C. Osthelder ${ }^{b}$, B.} Plaster $^{a}$, S. Slutsky ${ }^{b}$ and C. Swank ${ }^{b}$

a Department of Physics and Astronomy, University of Kentucky, Lexington, Kentucky 40506, U.S.A.

${ }^{b}$ Kellogg Radiation Laboratory, California Institute of Technology, Pasadena, California 91125, U.S.A.

E-mail: nima.nouri@uky.edu

\begin{abstract}
We present results from a first demonstration of a magnetic field monitoring system for a neutron electric dipole moment experiment. The system is designed to reconstruct the vector components of the magnetic field in the interior measurement region solely from exterior measurements.
\end{abstract}

KEYWORDS: .

${ }^{*}$ Corresponding author. 


\section{Contents}

1. Introduction 1)

2. Methodology 目

3. Experimental Apparatus and Procedure

田. Results

\section{Introduction}

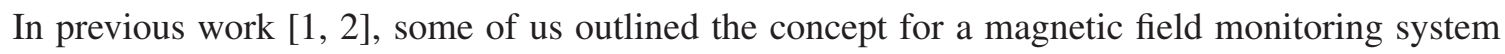
for a neutron electric dipole moment experiment in which the spatial dependence of the vector components of the magnetic field (and, hence, the gradients $\partial B_{i} / \partial x_{j}$ ) within the inaccessible interior measurement region are reconstructed solely from exterior measurements. In this paper, we show results from first prototyping tests of such a system. The results highlight the potential for the implementation of an improved system in an upcoming neutron electric dipole moment experiment to be carried out at the Spallation Neutron Source at Oak Ridge National Laboratory (i.e., the SNS nEDM experiment [3, 团). The work presented here complements techniques that have been developed to extract interior gradients from exterior measurements in scalar magnetometers [5], 6].

\section{Methodology}

Detailed discussions of the concepts underlying our field monitoring system were published previously [1], 2]. In brief, the basic idea is as follows. In a region of space containing no sources of current or magnetization (key assumptions underlying our concept), the magnetic scalar potential obeys the Laplace equation $\nabla^{2} \Phi_{M}(\vec{x})=0$, for which the solution can be written in spherical coordinates according to the well-known multipole expansion:

$$
\Phi_{M}(r, \theta, \phi)=\sum_{\ell=0}^{\infty} \sum_{m=0}^{+\ell} r^{\ell} P_{\ell}^{m}(\cos \theta)\left[a_{\ell m} \cos (m \phi)+b_{\ell m} \sin (m \phi)\right]
$$

Here, the $P_{\ell}^{m}(\cos \theta)$ denote the associated Legendre polynomials, and $a_{\ell m}$ and $b_{\ell m}$ denote $a$ priori unknown expansion coefficients. The magnetic field can then, of course, be written as $\vec{B}=$ $-\vec{\nabla} \Phi_{M}(\vec{x})$. Thus, if values for the magnetic field components $B_{i}$ can be determined at a number of exterior points, such measurements can be employed to determine values for the expansion coefficients $a_{\ell m}$ and $b_{\ell m}$, which in turn permits reconstruction of the scalar potential in the interior region (up to an arbitrary constant). Taking the gradient of the expansion equation (2.1) then uniquely 
Table 1. Sensitivity of the field components $\left(B_{x}, B_{y}, B_{z}\right)$ to the $a_{\ell m}$ and $b_{\ell m}$ expansion coefficients in terms of their associated basis functions, $f_{\ell m, i}(\vec{x})$ and $h_{\ell m, i}(\vec{x})$, in the expansion of the magnetic scalar potential. Some trivial basis functions are not listed, e.g., $f_{00, i}$, whose associated basis function in the scalar potential expression is a constant and thus does not appear in the expressions for the $B_{i}$. Also, expansion coefficients associated with basis functions which are linearly dependent on lower-order terms are redundant and do not need to be determined. Note that a ' 0 ' indicates that particular component offers no sensitivity to that particular expansion coefficient.

\begin{tabular}{lllllllllllll}
\hline & $\mathbf{a}_{\mathbf{1 0}}$ & $\mathbf{a}_{\mathbf{1 1}}$ & $\mathbf{b}_{\mathbf{1 1}}$ & $\mathbf{a}_{20}$ & $\mathbf{a}_{21}$ & $\mathbf{b}_{\mathbf{2 1}}$ & $\mathbf{b}_{\mathbf{2 2}}$ & $\mathbf{a}_{30}$ & $\mathbf{a}_{31}$ & $\mathbf{b}_{\mathbf{3 1}}$ & $\mathbf{b}_{\mathbf{3 2}}$ & $\mathbf{a}_{33}$ \\
\cline { 2 - 9 } $\mathbf{B}_{\mathbf{x}}$ & 0 & -1 & 0 & $f_{20, x}$ & $f_{21, x}$ & 0 & $h_{22, x}$ & $f_{30, x}$ & $f_{31, x}$ & $h_{31, x}$ & $h_{32, x}$ & $f_{33, x}$ \\
$\mathbf{B}_{\mathbf{y}}$ & 0 & 0 & -1 & $f_{20, y}$ & 0 & $h_{21, y}$ & $h_{22, y}$ & $f_{30, y}$ & $f_{31, y}$ & $h_{31, y}$ & $h_{32, y}$ & $f_{33, y}$ \\
$\mathbf{B}_{\mathbf{z}}$ & 1 & 0 & 0 & $f_{20, z}$ & $f_{21, z}$ & $h_{21, z}$ & 0 & $f_{30, z}$ & $f_{31, z}$ & $h_{31, z}$ & $h_{32, z}$ & 0 \\
\hline
\end{tabular}

determines the spatial dependence of the vector field components everywhere within the interior region.

Translating from spherical to rectangular coordinates, we write the components $B_{i}(\vec{x})$ in terms of the $a_{\ell m}$ and $b_{\ell m}$ expansion coefficients as

$$
B_{i}(\vec{x})=\sum_{\ell, m} a_{\ell m} f_{\ell m, i}(\vec{x})+b_{\ell m} h_{\ell m, i}(\vec{x}), \quad i \in\{x, y, z\} .
$$

where we use $f_{\ell m, i}(\vec{x})$ and $h_{\ell m, i}(\vec{x})$ to denote the basis functions in our expansion. Explicit expressions for $f_{\ell m, i}(\vec{x})$ and $h_{\ell m, i}(\vec{x})$ written in terms of $(x, y, z)$ rectangular coordinates (i.e., the coordinate system most compatible with typical experimental field measurements) are given in Appendix B of Ref. [2].

Table 1 summarizes the sensitivity of $\left(B_{x}, B_{y}, B_{z}\right)$ to the $a_{\ell m}$ and $b_{\ell m}$ expansion coefficients (up to $\ell=3$ ) in terms of these $f_{\ell m, i}$ and $h_{\ell m, i}$ basis functions. The point here is that measurements of $\left(B_{x}, B_{y}, B_{z}\right)$ provide (mostly) redundant information on the $a_{\ell m}$ and $b_{\ell m}$ expansion coefficients, as most of these coefficients are common to all three $\left(B_{x}, B_{y}, B_{z}\right)$ components; however, as can be seen in the table, certain coefficients can only be determined from measurements of a particular component (e.g., $b_{11}$ can only be determined from a measurement of $B_{y}$ ).

If one desires reconstruction of all the $\left(B_{x}, B_{y}, B_{z}\right)$ field components to a certain order (written in terms of the $a_{\ell m}$ and $b_{\ell m}$ coefficients up to that order), it is possible via measurements of any of the $\left(B_{x}, B_{y}, B_{z}\right)$ components providing appropriate sensitivity to these coefficients, with the number of required measurements equal to the number of $a_{\ell m}$ and $b_{\ell m}$ coefficients up to that order. As a specific example, suppose one desired to reconstruct $\left(B_{x}, B_{y}, B_{z}\right)$ to order $(\ell, m)=(3,3)$ in the scalar potential. As can be inferred from the table, such a reconstruction would require the determination of only 12 coefficients, $a_{10}$ through $a_{33}$, and, hence, only 12 exterior measurements. Five of these coefficients $\left(a_{20}, a_{30}, a_{31}, b_{31}, b_{32}\right)$ could be determined from a measurement of any of $\left(B_{x}, B_{y}, B_{z}\right)$ at any location in space where their respective basis functions are non-zero, four of these $\left(a_{21}, b_{21}, b_{22}, a_{33}\right)$ could be determined from any two of $\left(B_{x}, B_{y}, B_{z}\right)$, and three of these $\left(a_{10}, a_{11}, b_{11}\right)$ would require a measurement of a specific component (e.g., $a_{10}$ is sensitive only to $B_{z}$ ). Thus, one can optimize the choice of external measurements according to the needs of the par- 


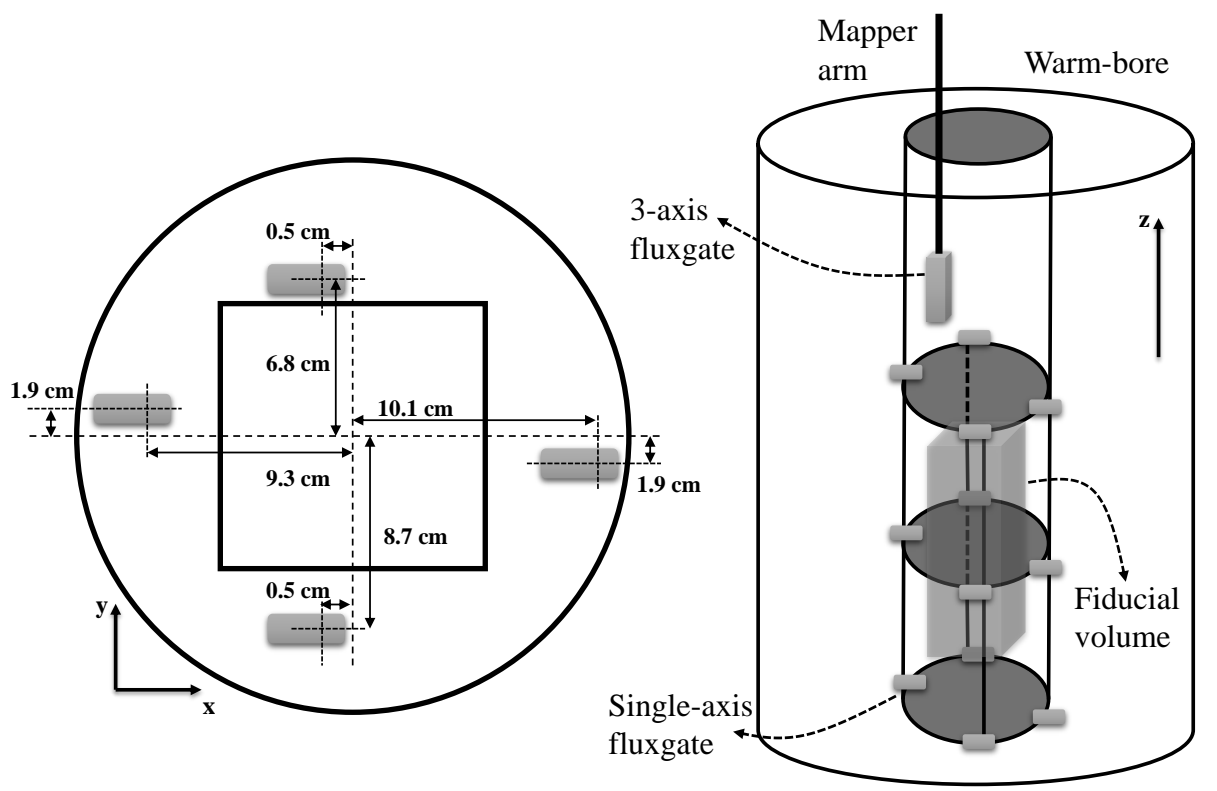

Figure 1. Left panel: A top view of the prototype array design. Right panel: Schematic figure of the experimental apparatus. A scaled version of the fiducial volume is also shown.

ticular experiment, with a careful choice of such providing for maximal information in the $(\ell, m)$ reconstruction from a minimal number of exterior measurements.

However, if the experiment is somehow constrained such that exterior measurements of only one of the field components is possible, then one can only reconstruct that particular component. For example, as can be seen in Table $\mathbb{1}, B_{x}$ does not provide sensitivity to $a_{10}, b_{11}$, and $b_{21}$, coefficients which would be needed for the reconstruction of $B_{y}$ and $B_{z}$. Continuing this example, with 12 exterior measurements of $B_{x}$, one could reconstruct $B_{x}$ up to a somewhat higher $(\ell, m)=(4,1)$ order, but would sacrifice the ability to reconstruct $B_{y}$ and $B_{z}$.

\section{Experimental Apparatus and Procedure}

A prototype field monitor system consisting of twelve single-axis fluxgate magnetometer probes was deployed within the magnetic field environment of an optimized $\cos \theta$ coil surrounded by multiple layers of magnetic shielding [7, 8] developed as a part of prototyping studies for the SNS nEDM experiment.

The single-axis fluxgate magnetometer probes, obtained from Stefan-Mayer Instruments [9], were mounted on a cylindrical-like support structure consisting of four aluminum rods, as shown in figure 1, with the cylindrical axis of this support structure oriented along the axis of the $\cos \theta \operatorname{coil}$. For the purposes of this first demonstration, the arrangement of the probes was rather simplistic; all of the probes were oriented along the $\cos \theta$ coil field direction (i.e., perpendicular to the axis of the $\cos \theta$ coil), with four probes mounted (identically) on each of three planes oriented, as shown in figure 1, perpendicular to the coil axis. 
To set the geometric scale for these first tests, note that the radius of this $\cos \theta$ coil is $32.4 \mathrm{~cm}$, with a length of $214.6 \mathrm{~cm}$ [7]. The effective radius of the support structure for the field monitor probes was $12.85 \mathrm{~cm}$, with the three planes separated by a distance of $30.5 \mathrm{~cm}$. During data taking, the $\cos \theta$ coil was energized to a nominal field of $\sim 220$ mGauss at the coil center. The readings in the twelve field monitor probes were then recorded simultaneously at one point in time. These twelve readings of the field component along the primary field direction, which we define to be $B_{x}$, were then fitted to the multipole expansion in equation (2.2) to determine the $a_{\ell m}$ and $b_{\ell m}$ coefficients up to order $(4,1)$, as discussed in the example given in the previous section. The magnetic field in the region interior to the prototype array was then calculated using these fitted coefficients. The accuracy of the reconstruction was then assessed by comparing the results of the reconstruction with explicit measurements of the field in the interior region carried out using a triple-axis fluxgate magnetometer probe [10] mounted to an automated magnetic mapper system. Note that the readings in the twelve field monitor probes were obtained prior to the measurements with the automated mapper system; we did not later average the monitor probes' readings over some period of time.

As is well known, a fluxgate magnetometer probe may read a non-zero value in a true "zero field" environment due to an offset associated with its geometry and its electronics. We extracted the offsets of each of our single-axis fluxgate magnetometer probes (relative to that of the triple-axis probe on the automated mapper system) via a procedure in which a large number (of order $\sim 700$ ) of readings from the mapper probe (conducted with the $\cos \theta$ coil de-energized) obtained in the interior region of the field monitor probes' support structure were fitted to a high-order multipole expansion. These fits were then extrapolated to the single-axis probes' locations, and any such differences between the extrapolated fitted values and the single-axis probes' actual readings were attributed to an offset.

\section{Results}

Results from our reconstruction of the interior magnetic field component $B_{x}$ (i.e., the component oriented along the $\cos \theta$ coil's primary field direction) along the three axes ( $z$-axis along the axis of the $\cos \theta$ coil's cylindrical support structure) are compared with direct measurements of the field obtained with the field mapper system in figure 2. Note that the reconstructed and measured field components $B_{x}$ were normalized to a value of 1.0 at the coil center. As can be seen there, the agreement is good, with the fractional gradient along the $x$-axis, $\left(\partial B_{x} / \partial x\right) / B_{x}$, on the level of $10^{-4}$ $\mathrm{cm}^{-1}$. Note that whereas the reconstructed fields were based on the readings obtained in the 12 single-axis probes at some instant in time, the measured fields (with the single triple-axis probe) were obtained over a time scale of order $\sim 1$ hour (due to the necessity of having to move the triple-axis probe to the different locations). Thus, the comparison between the reconstructed and measured fields may be degraded in the presence of time-varying background fields (which are not completely shielded), either on short time scales (e.g., between point-to-point measurements of the interior fields by the triple-axis probe), or on long time scales over the duration of the interior field measurements. To minimize the possibility of any such issues, the data were taken at night (data taking during the day was nearly impossible as a result of daytime operations of an overhead crane and delivery trucks) and the triple-axis probe was moved point-to-point by the automated 

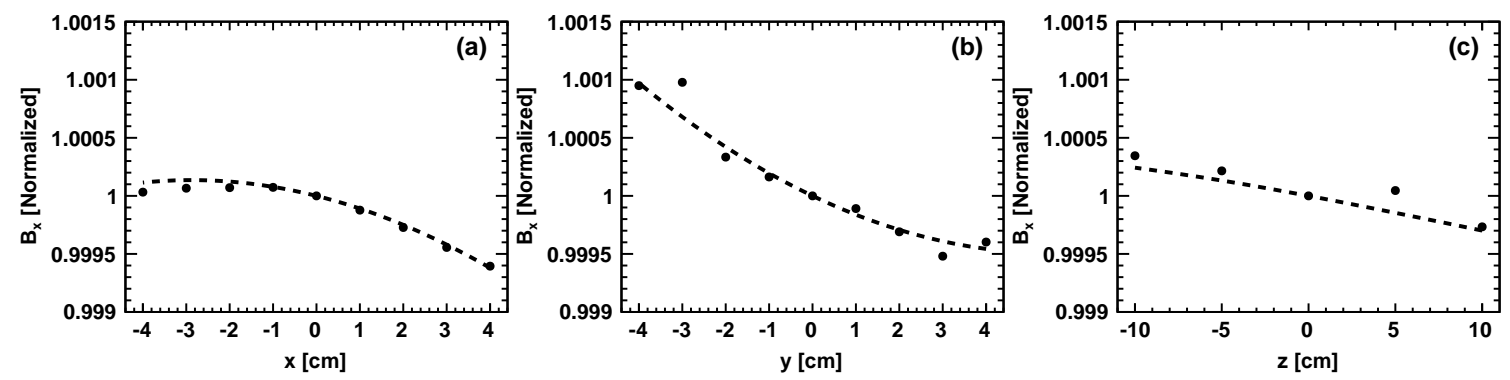

Figure 2. Comparison between the field component $B_{x}$ (i.e., along primary field direction) reconstructed from the exterior measurements in the field monitor array (dashed lines) and direct measurements obtained with the automated mapper system (dots). The three panels show results along the $x$-, $y$-, and $z$-axes. (Note that the error bars on the data points are smaller than the symbol size.)

mapper system in as expeditious manner as possible. Nevertheless, as can be seen in figure 2, some anomalies were observed, such as that at $y=-3 \mathrm{~cm}$.

Averaged over a volume corresponding to the SNS nEDM experiment's measurement volume (i.e., over a dense grid of points uniformly filling this volume, of which a subset are the data points along the central axes shown in figure 21), the reconstructed and measured fields agreed to better than $\sim 1.0 \%$. The reconstructed fractional gradients, $\left(\partial B_{x} / \partial x\right) / B_{x},\left(\partial B_{x} / \partial y\right) / B_{x}$, and $\left(\partial B_{x} / \partial z\right) / B_{x}$, averaged over this half-scale version of the measurement volume were $-1.1 \times 10^{-4}$ $\mathrm{cm}^{-1},-1.8 \times 10^{-4} \mathrm{~cm}^{-1}$, and $-0.3 \times 10^{-4} \mathrm{~cm}^{-1}$, respectively, which are to be compared to the direct measurements, which were $-1.4 \times 10^{-4} \mathrm{~cm}^{-1},-1.2 \times 10^{-4} \mathrm{~cm}^{-1}$, and $-0.1 \times 10^{-4} \mathrm{~cm}^{-1}$, again showing good agreement.

Improved results for the SNS nEDM experiment will ultimately be required. There, the goal will be to monitor the field gradients to the level of $10^{-5} \mathrm{~cm}^{-1}$ or better. Although this first prototype demonstrating our method does not yet meet this criterion, we are currently investigating the feasibility of employing vector field probes with smaller noise and offset characteristics, and in a next-version prototype we will also carry out a full optimization of the probe locations for maximal sensitivity to the successive higher-order $(\ell, m)$ terms in the multipole expansion.

\section{Acknowledgments}

This work was supported in part by the U. S. Department of Energy Office of Nuclear Physics under Award No. DE-FG02-08ER41557 and the National Science Foundation under Award No. 1205977. We are grateful to B. E. Allgeier for a careful reading of the manuscript.

\section{References}

[1] N. Nouri and B. Plaster, Sensitivity requirements for accessing interior magnetic field vector components in neutron electric dipole moment experiments via exterior boundary-value measurements, 2014 JINST 9 P11009. 
[2] N. Nouri and B. Plaster, Systematic optimization of exterior measurement locations for the determination of interior magnetic field vector components in inaccessible regions, Nucl. Instrum. Methods Phys. Res. A 767 (2014) 92.

[3] T. M. Ito, Plans for a Neutron EDM Experiment at SNS, J. Phys. Conf. Ser. 69 (2007) 012037.

[4] R. Golub and and S. K. Lamoreaux, Neutron electric-dipole moment, ultracold neutrons and polarized ${ }^{3} \mathrm{He}$, Phys. Rept. 237 (1994) 1.

[5] S. Afach et al., A measurement of the neutron to ${ }^{199} \mathrm{Hg}$ magnetic moment ratio, Phys. Lett. B 739 2014) 128.

[6] S. Afach et al., Measurement of a false electric dipole moment signal from ${ }^{199} \mathrm{Hg}$ atoms exposed to an inhomogeneous magnetic field, arXiv:1503.08651v2 [physics.atom-ph].

[7] A. Perez Galvan, et al, High uniformity magnetic coil for search of neutron electric dipole moment, Nucl. Instrum. Methods Phys. Res. A 6600 (2011) 147.

[8] S. Slutsky et al., to be published.

[9] Stefan-Mayer Instruments, www.stefan-mayer.com,. Their FLC 100 single-axis fluxgate magnetometer probe was employed for these studies.

[10] Bartington Instruments, www.bartington.com, The low-noise version of their Mag-03 triple-axis fluxgate magnetometer probe was employed for these studies. 